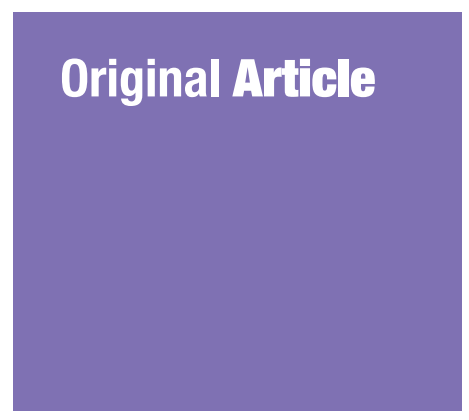

Submitted: 21Aug 2016 Accepted: 05 Jan 2017 Online: 14 Apr 2017

\section{A Combination of Trimethoprim- sulfamethoxazole and Ceftazidime Showed Good In Vitro Activity against Stenotrophomonas maltophilia}

\author{
Nabilah IsMAIL ${ }^{1}$, Zarifah ZAM ${ }^{2}$, Siti Asma HASSAN ${ }^{1}$, Zaidah AbduL \\ RAHMAN ${ }^{1}$
}
1 Department of Medical Microbiology and Parasitology, School of Medical Sciences, Universiti Sains Malaysia, 16150 Kubang Kerian, Kelantan, Malaysia
$2 \quad$ Hospital Taiping, Jalan Taming Sari, 34000 Taiping, Perak, Malaysia

To cite this article: Ismail N, Zam Z, Hassan SA, Abdul Rahman Z. A combination of trimethoprim-sulfamethoxazole and ceftazidime showed good in vitro activity against Stenotrophomonas maltophilia. Malays $J$ Med Sci. 2017;24(2):21-27. https://doi.org/10.21315/mjms2017.24.2.3

To link to this article: https://doi.org/10.21315/mjms2017.24.2.3

\title{
Abstract
}

Background: Stenotrophomonas maltophilia has emerged as an important nosocomial pathogen, capable of causing a wide spectrum of infections. Treatment is difficult because it is resistant to many antimicrobial agents, thus reducing the treatment options. The aims of this study were to describe the antimicrobial susceptibility patterns and synergistic effect of selected antimicrobial combinations against $S$. maltophilia isolates.

Methods: This was a descriptive cross-sectional study undertaken in the Hospital Universiti Sains Malaysia from April 2011 to March 2012. S. maltophilia isolated from various clinical specimens were included in the study. Antimicrobial susceptibility testing was done using the epsilometer test (E-test) and interpreted according to the guidelines of the Clinical and Laboratory Standards Institute. In the synergy test, the isolates were tested against six different antimicrobial combinations.

Results: In total, 84 S. maltophilia isolates were collected and analysed. According to the E-test, the antimicrobial susceptibility of trimethoprim-sulfamethoxazole (TMP-SMX), tigecycline, and ciprofloxacin was $100 \%, 91.1 \%$, and $88.9 \%$ respectively. The antimicrobial combination of TMP-SMX and ceftazidime showed the highest synergistic effect.

Conclusion: TMP-SMX remains the antimicrobial of choice to treat $S$. maltophilia infection. TMP-SMX and ceftazidime was the most effective combination in vitro.

Keywords: combination, trimethoprim-sulfamethoxazole, ceftazidime, Stenotrophomonas maltophilia

\section{Introduction}

Stenotrophomonas maltophilia is an aerobic, gram-negative, glucosenonfermentative, ubiquitous bacillus, which is found in a variety of environments and geographical regions, as well as inside and outside hospitals. The organism is of low virulence and considered an uncommon pathogen in immune-competent individuals (1). S. maltophilia has been described as an important causative agent of nosocomial infection (2). A previous study reported that bacteraemia and pneumonia were the two most common manifestations and that both were associated with high mortality (3).

Trimethoprim-sulfamethoxazole

(TMPSMX) remains the antimicrobial agent of choice to treat $S$. maltophilia infection, with susceptibility rates of $96 \%$ in most settings worldwide (4). However, resistance towards TMP-SMX is an increasing problem. Although 
the resistance rate was reported to vary, depending on the institution and geographic area, it was generally less than $10 \%(5,6)$. In our study setting at Hospital Universiti Sains Malaysia (HUSM), based on five-year data from 2006 to $2010,7.14 \%$ of $S$. maltophilia isolates were resistant to TMP-SMX (unpublished data).

The treatment of $S$. maltophilia infections is often problematic because the pathogen shows intrinsic resistance to many antimicrobial agents, including carbapenems, aminoglycosides, antipseudomonal penicillins, and antipseudomonal third-generation cephalosporins (7). In view of the limited antimicrobials available for treatment, this study aimed to determine the antimicrobial susceptibility pattern of $S$. maltophilia isolates in HUSM and to determine the antimicrobial synergistic effect of potential antimicrobial combinations.

\section{Materials and Methods}

\section{Sample collection}

This was a cross-sectional study undertaken in the HUSM from April 2011 until March 2012. $S$. maltophilia isolates obtained from various clinical specimens (sputum, endotracheal aspirates, body fluid, female genitals, pus, tissue, cerebrospinal fluid, urine, and blood) were included in the study. Repeated specimens from the same patient within three weeks were excluded.

\section{Antimicrobial susceptibility testing}

All the isolates were tested against a panel of antimicrobial agents, including TMP-SMX, ceftazidime, ciprofloxacin, and imipenem, based on the guidelines of the Clinical and Laboratory Standards Institute (CLSI) (8). Piperacillintazobactam and tigecycline were also included in the panel for antimicrobial susceptibility testing (AST). AST was performed using the epsilometer test (E-test) (BioMerieux SA, MarcyI'Etoile, France), according to the manufacturer's instructions. The isolates were cultured on Mueller-Hinton agar (Thermo Scientific ${ }^{\mathrm{TM}}$ Remel Muller Hinton, USA) for AST and incubated at $35{ }^{\circ} \mathrm{C}$ for 18 hours-24 hours. The AST results for TMP-SMX were interpreted according to the standards of the CLSI (9). AST of piperacillintazobactam, ceftazidime, ciprofloxacin, and imipenem were interpreted using breakpoints for Pseudomonas aeruginosa, according to the CLSI's guideline (9). AST of tigecycline, which is not included in the CLSI guideline, was interpreted using other published protocols (10, 11).

\section{Synergy testing}

Using the E-test method, the isolates were subjected to antimicrobial synergy testing against six selected combinations of antimicrobial agents: TMP-SMX plus colistin, TMP-SMX plus ceftazidime, TMP-SMX plus sulperazone, ciprofloxacin plus piperacillintazobactam, TMP-SMX plus piperacillintazobactam, and TMP-SMX plus rifampicin. The combinations were selected based on the availability of the antimicrobial agents in the study setting and the lack of previous studies on these combinations. E-test strips were placed on Mueller-Hinton agar medium in a cross formation, with a $90^{\circ}$ angle at the intersection between the scales at the respective minimal inhibitory concentrations (MICs) for both antimicrobial agents (Figure 1), as previously described (12). Cultured plates were placed inversely for incubation at $37{ }^{\circ} \mathrm{C}$ for 16 hours -18 hours (12).

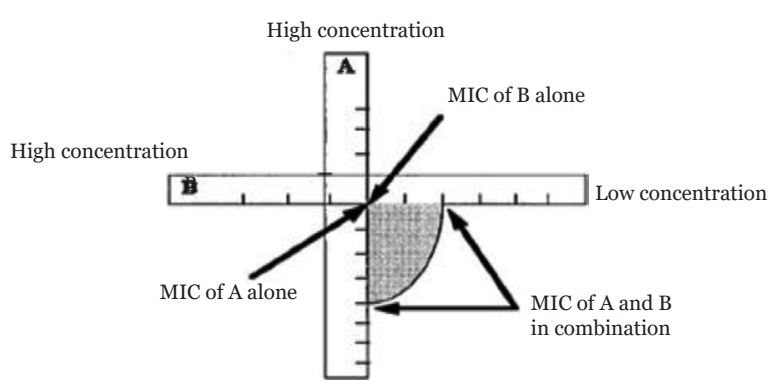

Figure 1. Diagrammatic illustration of E-test strips placement for synergy test, adopted from White et al., 1996 (12).

The results of the synergy testing were interpreted as synergistic, antagonism, or indifference (13). Synergism refers to a positive interaction, in which the combined effect of the test drugs is significantly greater after combination than the sum of both effects (14). Antagonism refers to negative interaction, in which the combined effect of the test drugs is significantly less than their independent effects when they are tested separately (14). Indifference denotes a combined action, in which the effect of the test drugs combined is no greater than the effect when used alone (14).

After incubation, the MICs were interpreted at the point of intersection between the inhibition zone and E-test strip (Figure 2). The calculation and interpretation of the results 
of the synergy test were done according to a previously published protocol (13). Briefly, synergy was defined as $\Sigma$ FIC $<0.5$; antagonism was defined as $\Sigma$ FIC $>4$, and indifference was defined as $\Sigma$ FIC $>0.5$ and $\Sigma$ FIC $<4$.

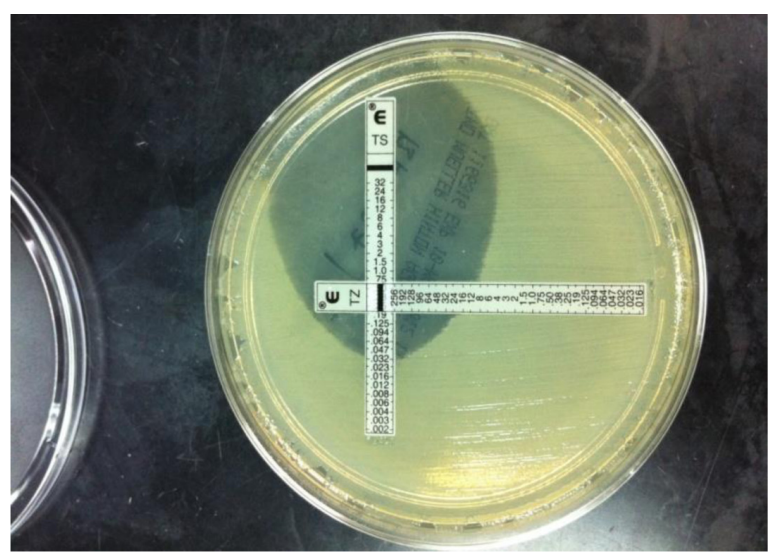

Figure 2. Photograph of E-test result for synergy test observed in TMP-SMX and ceftazidime combination.

\section{Results}

In total, 84 S. maltophilia isolates were collected during the study period. Of those, $57 \%(n=48)$ were isolated from endotracheal aspirate and other body fluid, and 22\% $(n=18)$ were isolated from blood. The other origins of the isolates were as follows: pus and tissue (12\%, $n=10$ ), sputum ( $7 \%, n=6)$, and cerebrospinal fluid and eye specimens (o.8\%, $n=1$ for each). All the isolates were susceptible to TMPSMX and resistant to imipenem. The majority of the isolates showed high susceptibility to ciprofloxacin and tigecycline (Table 1).
Table 1. Susceptibility rate of $S$. maltophilia isolates using E-test

\begin{tabular}{lc}
\multicolumn{1}{c}{ Antimicrobials } & $\boldsymbol{n}(\%)$ \\
Trimethoprim-sulfamethoxazole & $45(100)$ \\
Ciprofloxacin & $40(88.9)$ \\
Piperacillin-tazobactam & $9(20)$ \\
Ceftazidime & $26(57.8)$ \\
Tigecycline & $41(91.1)$ \\
Imipenem & o (o) \\
\hline
\end{tabular}

The synergistic effects of the six antimicrobial combinations were tested. Synergism was observed in four sets of the antimicrobial combinations, with the highest rate observed with TMP-SMX plus ceftazidime, followed by TMP-SMX plus sulperazone, ciprofloxacin plus piperacillin-tazobactam, and TMP-SMX plus piperacillin-tazobactam (Figure 3). The isolates showed indifference results for all antimicrobial combination at various percentages and no antagonism was seen.

\section{Discussion}

S. maltophilia can cause serious infection in severely immunocompromised and debilitated individuals. The infection continues to pose a therapeutic challenge because of the emergence of resistant strains, causing treatment failure. $S$. maltophilia is consistently susceptible to TMPSMX, making it the drug of choice (4). However, recent data reported the emergence of a strain resistant to TMP-SMX, further complicating the management of patients $(5,6)$.

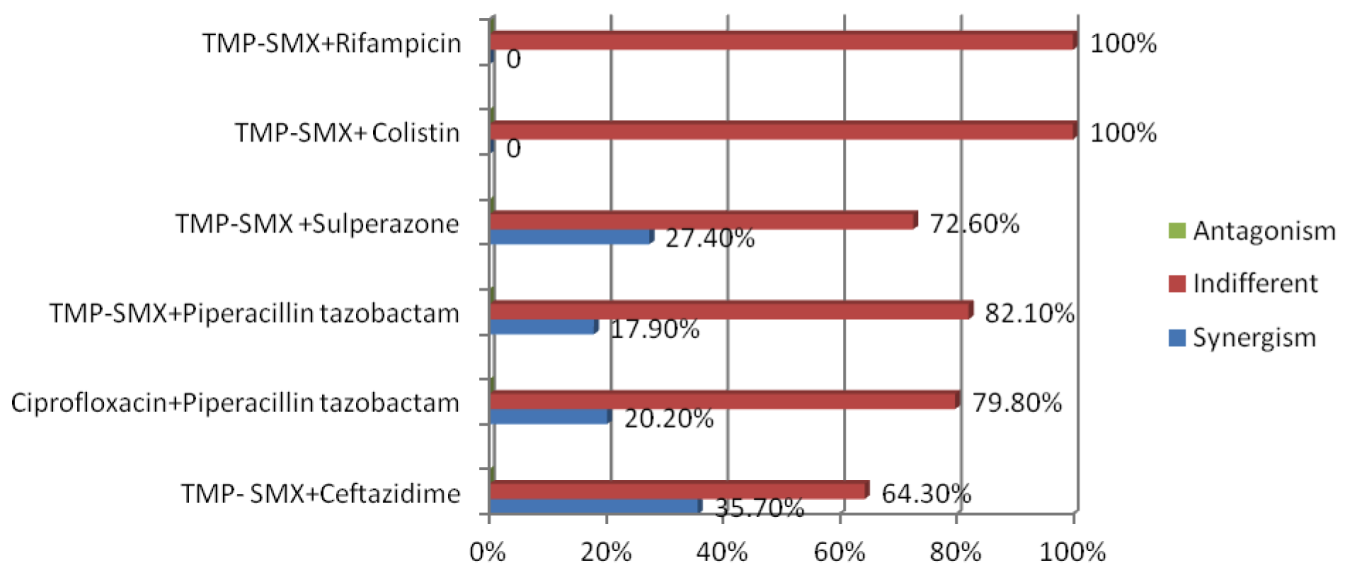

Figure 3. Synergistic activity of antimicrobial combination against $S$. maltophilia isolates 
In the present study, all the isolates tested with the E-test were susceptible to TMP-SMX. Thus, this can still be considered the antimicrobial agent of choice to treat $S$. maltophilia infection in the study setting. Despite the good susceptibility to TMPSXT, a previous study reported that TMPSMX alone was bacteriostatic at best against all isolates of $S$. maltophilia and that all antimicrobial combinations were more active than monotherapy, as determined by bacterial reductions at both $24 \mathrm{~h}$ and $48 \mathrm{~h}(P<0.0001)$ (15).

The results of the present showed that the most effective antimicrobial agents against $S$. maltophilia were TMP-SMX, followed by tigecycline, ciprofloxacin, and ceftazidime. Piperacillin-tazobactam exhibited a high rate of resistance rate towards $S$. maltophilia isolates. These findings are in line with those of previous research (16). However, they are in contrast to those of another study, which reported higher susceptibility rates of piperacillin-tazobactam in a disc diffusion test and E-test $(61.5 \%$ and $46.2 \%$, respectively) (17). Tigecycline was not tested in the latter study. However, previous studies showed that tigecycline was one of the most promising therapeutic options for treating multidrug-resistant Acinetobacter and $S$. maltophilia infections $(11,18)$. In common with the findings of a previous study (16), tigecycline showed good in vitro activity against S. maltophilia in the present study.

Fluoroquinolones are one of the main alternatives to TMP-SMX for the treatment of $S$. maltophilia infections (19). The findings of the present study correlated well with those of other studies, which suggested that fluoroquinolones, such as ciprofloxacin, had potent in vitro activity against $S$. maltophilia $(20,21)$. In the current study, the susceptibility rate to ciprofloxacin was $88.9 \%$, which was comparable with that found in a previous study, which reported a susceptibility rate of $82.4 \%$ (22). However, several studies demonstrated poor activity of ciprofloxacin against $S$. maltophilia, with susceptibility rates, on average, lower than $50 \%$ $(23,24)$. Thus, the choice of antimicrobial agent to treat $S$. maltophilia infection depends on local susceptibility patterns.

Increasing numbers of reports of resistance to TMP-SMX have fuelled studies of antimicrobial combinations $(5,15,16)$. One of the aims of antimicrobial combinations is to enhance the synergistic activity and bactericidal effect of particular antimicrobial agents against specific organisms. Theoretically, antimicrobial combinations can also delay the emergence of microbial mutants resistant to one drug in chronic infections by the use of second or third non-cross reacting drugs (14). As shown in the present study, the TMP-SMX-ceftazidime combination exhibited the highest rate of synergism. A higher synergistic rate of $72 \%$ was reported by a previous study (16). Our findings also correlated with another study that reported net bacterial kill of the isolates and provided significant benefit over monotherapy (15).

However, the combination of TMP-SMXcolistin failed to exhibit a synergistic effect. This finding was contradictory to that of earlier studies, which detected TMP-SMX-colistin synergy in $41.7 \%$ (25) and $32 \%$ (16) of isolates. The discrepancy in the results could be due to the use of different methods (time-kill assay) in the previous studies. For the combination of ciprofloxacin and piperacillin-tazobactam, a synergistic effect was observed in $20.2 \%$ of the isolates, as compared to $32 \%$ reported in an earlier study (16). Several reports proposed the use of a combination of TMP-SMX plus ticarcillin-clavulanic acid $(16,26,27)$. However, this combination was not included in the present study because ticarcillin-clavulanic acid is not available in the study setting.

Although the choice of monotherapy or combination therapy for $S$. maltophilia infection remains a matter of debate, several studies suggested the use of antimicrobial combination treatment, especially in patients who are at risk of $S$. maltophilia infection $(21,27)$. Other potential uses for antimicrobial combination therapy include patients with resistant organisms, sulfonamide-intolerant patients, and neutropenic patients (28).

An in vitro method of detecting synergy, which is simple to perform, accurate, reproducible, and has the potential for clinical extrapolation, is highly desirable. Both the timekill assay and checkerboard methods using broth dilution are widely used to assess synergy, but they are time consuming and labour intensive (12). Today, the E-test is more practical in routine laboratory testing and is a reliable alternative to broth dilution (16). A previous study reported that confirmation of the E-test synergy results by microdilution checkerboard method revealed agreement for TMP-SXT plus ceftazidime combination against $S$. maltophilia isolates (16). 
In conclusion, TMP-SMX remains the antimicrobial agent of choice to treat $S$. maltophilia infection in the study setting. The TMP-SMX-ceftazidime combination should be considered when monotherapy fails to combat $S$. maltophilia infections. Other antimicrobial combinations merit further evaluation to determine their synergistic action.

\section{Ethical approval}

The study was approved by the Human Research Ethics Committee of the Universiti Sains Malaysia (USMKK/PPP/JEPeM. 237.4 (2.4)) prior to conducting the study.

\section{Acknowledgement}

We gratefully acknowledge the staff in the Department of Medical Microbiology and Parasitology, Universiti Sains Malaysia, especially the assistance of Puan Rosliza Abd Rahman and Puan Fadzilah Yusof. We also thank Dr Aniza bt Abd Aziz, a statistician in the Universiti Sultan Zainal Abidin, Terengganu, and Puan Anis Kausar, a statistics officer in the IPS unit of the Universiti Sains Malaysia, for their help with the statistical part of the study.

\section{Fund}

The study was funded by the Universiti Sains Malaysia in the form of a short-term grant (304/PPSP/61311069).

\section{Conflict of Interest}

The authors declare that they have no competing interests.

\section{Authors' Contributions}

Conception and design: NI, ZZ

Analysis and interpretation of the data: ZZ, SAH, ZAR Drafting of the article: NI, ZZ

Critical revision of the article for important intellectual content: NI, SAH, ZAR

Final approval of the article: NI, SAH, ZAR

Provision of study materials or patients: NI

Statistical expertise: ZZ

Obtaining of funding: NI

Administrative, technical, or logistic support: NI, ZZ

Collection and assembly of data:ZZ

\section{Correspondence}

Dr Nabilah Ismail

MD, MPath (Microbiology)

Department of Medical Microbiology and Parasitology, School of Medical Sciences, Universiti Sains Malaysia, 16150 Kubang Kerian,

Kelantan, Malaysia

Tel: +60139233635

Fax: 6097676289

E-mail:drnabilah@usm.my

\section{References}

1. Denton M, Kerr KG. Microbiological and clinical aspects of infection associated with Stenotrophomonas maltophilia. Clin Microbiol Rev. 1998;11(1):57-80.

2. Aisenberg G, Rolston KV, Dickey BF. Stenotrophomonas maltophilia pneumonia in cancer patients without traditional risk factors for infection, 1997-2004. Eur J Clin Microbiol Infect. Dis. 2007;26(1):13-20. https://doi.org/10.1007/ s10096-006-0243-7

3. Cho SY, Lee DG, Choi SM, Park C, Chun HS, Park YJ, et al. Stenotrophomonas maltophilia bloodstream infection in patients with hematologic malignancies: a retrospective study and in vitro activities of antimicrobial combinations. BMC Infect Dis. 2015;15:69. https://doi.org/10.1186/s12879-015-0801-7

4. Farrell DJ, Sader HS, Jones RN. Antimicrobial susceptibilities of a worldwide collection of Stenotrophomonas maltophilia isolates tested against tigecycline and agents commonly used for S. maltophilia infections. Antimicrob Agents Chemother. 2010;54(6):2735-2737. https://doi. org/10.1128/AAC.01774-09

5. Chung HS, Hong SG, Kim YR, Shin KS, Whang $\mathrm{DH}$, Ahn JY, et al. Antimicrobial susceptibility of Stenotrophomonas maltophilia isolates from Korea, and the activity of antimicrobial combinations against the isolates. J. Korean Med. Sci. 2013;28(1):62-66. https://doi.org/10.3346/ jkms.2013.28.1.62 
6. Gales AC, Jones RN, Forward KR, Linares J, Sader HS, Verhoef J. Emerging importance of multidrug-resistant Acinetobacter species and Stenotrophomonas maltophilia as pathogens in seriously ill patients: geographic patterns, epidemiological features, and trends in the SENTRY Antimicrobial Surveillance Program (1997-1999). Clin. Infect. Dis. 2001;32(Suppl. 2):104-113. https://doi.org/10.1086/320183

7. Nicodemo AC, Araujo MRE, Ruiz AS, Gales AC. In vitro susceptibility of Stenotrophomonas maltophilia isolates: comparison of disc diffusion, E-test and agar dilution methods. J Antimicrob Chemother. 2004;53(4):604-608. https://doi. org/10.1093/jac/dkh128

8. The Clinical and Laboratory Standards Institute (CLSI). Methods for dilution antimicrobial susceptibility tests for bacteria that grow aerobically; approved standards-tenth edition. CLSI document Mo7-A10. Wayne, PA: Clinical and Laboratory Standards Institute; 2015.

9. The Clinical and Laboratory Standards Institute (CLSI). Performance Standards for Antimicrobial Susceptibility Testing. 26th ed. CLSI Supplement M1ooS. Wayne, PA: Clinical and Laboratory Standard Institute; 2016.

10. Jones RN, Ferraro MJ, Reller LB, Schreckenberger PC, Swenson JM, Sader HS. Multicenter studies of Tigecycline disc diffusion susceptibility results for Acinetobacter spp. J Clin Microbiol. 2007;45(1):227-230. https://doi. org/10.1128/JCM.01588-06

11. Insa R, Cercenado E, Goyanes MJ, Morente A, Bouza E. In vitro activity of tigecycline against clinical isolates of Acinetobacter baumannii and Stenotrophomonas maltophilia. $J$ Antimicrob Chemother. 2007;59(3):583-585. https://doi. org/10.1093/jac/dkl496

12. White RL, Burgess DS, Manduru M, Bosso JA. Comparison of three different in vitro methods of detecting synergy: time-kill, checkerboard and E-test. Antimicrob Agents Chemother. 1996;40(8):1914-1918.

13. Satish KP, MR, Eliopulos GM. Antimicrobial combinations. In: Lorien V, editor. Antibiotics in Laboratory Medicine. Philadelphia: Lippincott Williams and Wilkins. 2005; 365-440.

14. Jawetz, Melnick \& Adelberg's. Medical Microbiology. 27th ed. Lange: McGraw-Hill Education; 2016.
15. Zelenitsky SA, Iacovides H, Ariano RE, Harding GK. Antimicrobial combinations significantly more active than monotherapy in an in vitro infection model of Stenotrophomonas maltophilia. Diagn Microbiol Infect Dis. 2005;51:39-49.

16. Gulmez D, Cakar A, Sener B, Karakaya J, Hascelik G. Comparison of different antimicrobial susceptibility testing methods for Stenotrophomonas maltophilia and results of synergy testing. $J$ Infect Chemother. 2010;16:322-328. https://doi.org/10.1007/ s10156-010-0068-2

17. Tatman-Otkun M, Gürcan S, Özer B, Aydoslu B and Bukavaz S. The antimicrobial susceptibility of Stenotrophomonas maltophilia isolates using three different methods and their genetic relatedness. BMC Microbiol. 2005;5:24. https:// doi.org/10.1186/1471-2180-5-24

18. Hoban DJ, Bouchillon SK, Johnson BM, Johnson JL, Dowzicky MJ. In vitro activity of tigecycline against 6792 gram-negative and gram-positive clinical isolates from the global tigecycline evaluation and surveillance trial (TEST Program, 2004). Diagn Microbiol Infect Dis. 2005;52:215227.

19. Nicodemo AC, Garcia Paez JI. Antimicrobial therapy for Stenotrophomonas maltophilia infections. Eur $J$ Clin Microbiol Infectious Dis. 2007;26:229-237.

20. Looney WJ, Narita M, Muhlemann K. Stenotrophomonas maltophilia: an emerging opportunist human pathogen. Lancet Infect Dis. 2009;9:312-323.

21. Falagas ME, Valkimadi PE, Huang YT, Matthaiou DK, Hsueh PR. Therapeutic options for Stenotrophomonas maltophilia infections beyond co-trimoxazole: a systematic review. J Antimicrob Chemother. 2008;62:889-894.

22. Samonis G, Karageorgopoulos DE, Maraki S, Levis P, Dimopoulou D, Spernovasilis NA, et al. Stenotrophomonas maltophilia infections in a general hospital: patient characteristics, antimicrobial susceptibility, and treatment outcome. PLoS ONE. 2012;7(5):e37375. https:// doi.org/10.1371/journal.pone.0037375. 
Original Article |Stenotrophomonas maltophilia combination therapy

23. Jones RN, Sader HS, Beach ML. Contemporary in vitro spectrum of activity summary for antimicrobial agents tested against 18569 strains non-fermentative Gram-negative bacilli isolated in the SENTRY Antimicrobial Surveillance Program (1997-2001). Int J Antimicrob Agents. 2003;22:551-556. https://doi.org/10.1016/ So924-8579(03)00245-0

24. Sader HS, Jones RN, Gales AC, Silva JB, Pignatari AC and SENTRY Participants Group (Latin America). SENTRY antimicrobial surveillance program report: Latin American and Brazilian results for 1997 through 2001. Braz J Infect Dis. 2004;8:25-79. https://doi.org/10.1590/S141386702004000100004

25. Giamarellos-Bourboulis EJ, Karnesis L, Giamarellou H. Synergy of colistin with rifampin and trimethoprim/sulfamethoxazole on multidrug-resistant Stenotrophomonas maltophilia. Diagn Microbiol Infect Dis. 2002;44:259-260.
26. Poulos CD, Matsumura SO, Willey BM, Low DE, McGeer A. In vitro activities of antimicrobial combinations against Stenotrophomonas (Xanthomonas) maltophilia. Antimicrob Agents Chemother. 1995;39(10):2220-2223.

27. Liaw SJ, Teng LJ, Hsueh PR, Ho SW, Luh KT. In vitro activities of antimicrobial combinations against clinical isolates of Stenotrophomonas maltophilia. $J$ Formos Med Assoc. 2002;101:495-501.

28. Krueger TS, Clark EA, Nix DE. In vitro susceptibility of Stenotrophomonas maltophilia to various antimicrobial combinations. Diagn Microbiol and Infect Dis. 2001;41:71-78. https:// doi.org/10.1016/So732-8893(01)00281-4 